\title{
eJRIEPS
}

Ejournal de la recherche sur l'intervention en éducation physique et sport

Numéro spécial 2 | 2019

Actes du congrès ARIS 2016

\section{Analyse de la transition entre l'enseignement secondaire et l'enseignement supérieur chez les sportifs de haut niveau en Fédération Wallonie- Bruxelles}

Analysis of the transition between secondary school and higher education in top level athletes in Wallonia-Brussels Federation

Catherine Theunissen, Nathan Bolaers et Marc Cloes

\section{OpenEdition}

Journals

Édition électronique

URL : http://journals.openedition.org/ejrieps/969

DOI : 10.4000 /ejrieps.969

ISSN : 2105-0821

Éditeur

ELLIADD

\section{Référence électronique}

Catherine Theunissen, Nathan Bolaers et Marc Cloes, « Analyse de la transition entre l'enseignement secondaire et l'enseignement supérieur chez les sportifs de haut niveau en Fédération WallonieBruxelles », eJRIEPS [En ligne], Numéro spécial 2 | 2019, mis en ligne le 01 juillet 2019, consulté le 21 novembre 2019. URL : http://journals.openedition.org/ejrieps/969; DOI : 10.4000/ejrieps.969

La revue eJRIEPS est mise à disposition selon les termes de la Creative Commons Attribution 4.0 International License. 


\section{Analyse de la transition entre l'enseignement secondaire et l'enseignement supérieur chez les sportifs de haut niveau en Fédération Wallonie-Bruxelles}

Theunissen Catherine, Bolaers Nathan \& Cloes Marc

Département des Sciences de la Motricité - Service d'Intervention et Gestion des APS (SIGAPS). Université de Liège, Belgique.

\section{Résumé}

La transition scolaire entre les études secondaires (l'équivalent des lycées en France) et supérieures représente une période charnière pour les sportifs de haut niveau qui sont engagés dans un double projet de vie, sportif et scolaire. La présente étude analyse les opinions, à propos de cette période critique, de 103 jeunes, reconnus officiellement par la Fédération Wallonie-Bruxelles (Belgique francophone) comme espoirs sportifs. Les résultats montrent que $11 \%$ ont abandonné leur pratique sportive pour se consacrer à leurs études supérieures ; $73 \%$ pensent que les aménagements sont mieux organisés dans le secondaire que dans le supérieur, avec de fortes disparités en fonction des établissements de formation. Étonnamment, $43 \%$ mentionnent ne pas avoir reçu d'informations concernant les aménagements possibles pour les sportifs de haut niveau. Après cette année de transition, une diminution de $27 \%$ du nombre de jeunes ayant bénéficié du statut spécial octroyé par le Ministère des sports est également relevée. Un projet basé sur la continuité des démarches et des aménagements semble nécessaire pour aider ces étudiants-sportifs à mener à bien leur double vie.

Mots clés : Sportifs de haut niveau, carrière sportive, parcours scolaire, transition scolaire, aménagements. 
Analysis of the transition between secondary school and higher education in top level athletes in Wallonia-Brussels Federation

\section{Summary}

The transition between the secondary school and higher education represents a pivotal period for the top-level athletes who make a commitment in a double life plan: sports and school. The present study analyzes the opinions of 103 young people, officially recognized as promising athletes by the Federation Wallonia-Brussels (Belgium), about this critical period. The results showed that $11 \%$ abandoned their sports practice to engage themselves in their studies; $73 \%$ believed that the arrangements are better organized in secondary school level than in higher education, with strong disparities according to the institutions. Strangely, 43\% mentioned that they did not receive information concerning the possible arrangements for the top-level athletes. After this year of transition a decrease of $27 \%$ of the number of athletes who obtained the special status granted by the Ministry of sports, was also observed. A project based on the continuity of the procedures and of the arrangements seems necessary to help these students-athletes to achieve their double life.

Key words: Top level athletes, sports career, academic preparation, school transition, arrangements

\section{Introduction}

Vivre de la pratique de son sport constitue un véritable défi. Aussi performant soit-il, il est difficile pour un sportif de se projeter dans l'avenir et s'imaginer vivre des gains récoltés au cours de ses prestations. En effet, tous ses rêves peuvent s'effondrer brusquement à la suite d'une blessure ou d'une contre-performance. La majorité des jeunes sportifs opte donc à présent pour un double projet de vie, à la fois scolaire et sportif. Habituellement, la transition scolaire (secondaire-supérieur) coïncide avec une intensification des entraînements et une difficulté scolaire accrue. Le ressenti des sportifs concernés nous paraît essentiel pour évaluer l'efficacité des aménagements proposés par les différentes institutions scolaires et l'Administration Générale du Sport (AG Sport). 


\section{eJRIEPS Spécial 2 (juillet 2019)}

\section{Revue de littérature}

En Belgique francophone, une impulsion a été donnée au début des années 2000 à la double carrière des sportifs de haut niveau, à la suite d'un changement de politique au niveau de la Fédération Wallonie-Bruxelles (AG Sport, nd). En effet, pendant une soixantaine d'années, la politique sportive belge se voulait promotrice de la santé et s'orientait vers la jeunesse. Le fossé avec le sport de haut niveau avait donc tendance à se creuser. Les maigres performances belges aux Jeux Olympiques avant Rio 2016 et l'argent investi dans le sport en Belgique témoignent de ce constat puisque seul 0,01\% du budget total était attribué au sport en Belgique francophone alors qu'il atteint en France, en Hollande et en Suède, entre 0,07 et 0,08\%. L'organisation complexe du sport en Belgique semble à la base des faibles moyens financiers investis. En effet, en Belgique, le sport est une compétence communautarisée. Le sport francophone est ainsi sous la tutelle de la Fédération Wallonie-Bruxelles en ce qui concerne les compétences liées aux personnes (formation des cadres, suivi des sportifs de haut niveau, ...) et de deux régions (wallonne ou bruxelloise) pour tout ce qui concerne les biens (infrastructures) - (Cloes, 2011). Cette situation complexe engendre une difficulté à définir une politique cohérente et orientée vers le long terme.

En matière de politique sportive, l'étude SPLISS (Sports Policy Factors Leading to International Succes), réalisée par De Bosscher, De Knop, van Bottenburg, Bingham et Shilbi (2006), a permis d'identifier neuf piliers conditionnant les performances sportives d'un état :

- L'approche intégrée de la politique de développement ;

- La pratique du sport ;

- Le soutien financier ;

- Le système de détection et de développement des talents ;

- Le soutien aux athlètes pendant et après leur carrière sportive ;

- L'offre de coaching et le développement des entraîneurs ;

- La compétition internationale ;

- La recherche scientifique ;

- Les infrastructures sportives.

Les conclusions de cette recherche qui compare les résultats de cinq pays avec la Fédération Wallonie-Bruxelles soulignent que cette dernière atteignait la moyenne pour un seul des piliers : la pratique du sport. Ce constat a clairement démontré les déficiences de la politique sportive de haut niveau dans la partie francophone de la Belgique. 


\section{eJRIEPS Spécial 2 (juillet 2019)}

Depuis 2004, le gouvernement de la Fédération Wallonie-Bruxelles a changé de ligne de conduite et opté pour une politique double orientée à la fois «sport pour tous » et «sport de haut niveau ». Parallèlement à cela, le gouvernement a la volonté d'améliorer la détection des jeunes talents en développant les sport-études et en améliorant les statuts particuliers attribués aux espoirs et athlètes de haut niveau. C'est par l'intermédiaire de la cellule «Projet de vie » de l'AG Sport que ces différents aspects se mettent en œuvre.

Afin de garantir la reconversion des sportifs de haut niveau en fin de carrière, un projet à la fois professionnel et scolaire s'avère indispensable. Pour parvenir à combiner les deux projets, de nombreux pays ont opté pour la mise en place de différentes stratégies. Les aides diffèrent toutefois fortement d'un pays à l'autre.

En Amérique du nord, le concept d'étudiant sportif existe depuis longtemps et le prestige de certains sports engendre régulièrement une mise en avant plus importante de la réussite sportive par rapport à la réussite scolaire. C'est la raison pour laquelle la NCAA (National Collegiate Athletics Association - http://www.ncaa.org) a tenté d'atténuer cette dérive en interdisant aux étudiants de première année universitaire de participer à des compétitions sportives et en limitant leurs prestations à des entraînements.

En France, c'est la Commission du Sport de Haut Niveau (CSHN http://www.sports.gouv.fr/pratiques-sportives/sport-performance/) qui définit les orientations de la politique nationale à ce sujet. Les directeurs techniques fédéraux placent les candidats potentiels sur une liste. C'est ensuite le ministère qui entérine les candidatures approuvées. Ainsi, les sportifs " élites » doivent avoir réalisé une performance lors d'une compétition internationale, les sportifs « jeunes » représentent des athlètes en devenir et les sportifs « seniors » ont remporté un titre international durant l'année en cours. Une dernière catégorie est celle des sportifs « en reconversion».

La France compte également sur un système de pôles. Ceux-ci ont trois grandes priorités : (1) la préparation sportive, (2) la formation scolaire, universitaire ou professionnelle et (3) le suivi personnalisé des sportifs. Deux types de pôles sont à distinguer : les pôles « Espoirs » et les pôles "France". Les sportifs qui intègrent ces pôles bénéficient idéalement d'aménagements tels que la mise en ligne de cours à distance, des outils d'autoapprentissage, des cours téléphoniques, la découverte des métiers, l'accompagnement individuel, ...

L'Association Sport-Étude mise en place en Suisse (ASE - http://sportetude.ecolevs.ch/concept.asp?inside=concept) se charge d'assurer la coordination entre les différents partenaires nécessairement impliqués (parents, clubs, médecins, entraîneurs, 


\section{eJRIEPS Spécial 2 (juillet 2019)}

...). Le modèle illustré par Hug (2003 - figure 1) présente les principales influences environnementales susceptibles d'exercer un impact sur les sportifs de haut niveau en fonction des caractéristiques du contexte socio-économique propre à la discipline sportive et aux choix des pouvoirs publics.

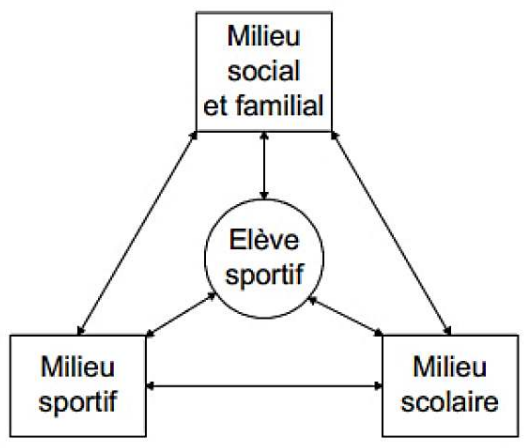

Figure 1 : système relationnel idéal de l'élève-sportif, d'après Hug (2003)

La Belgique francophone porte également un intérêt envers ces sportifs puisqu'un arrêté du gouvernement datant du 11 mars 2010 définit les conditions d'obtention des statuts. II en existe trois : (1) les sportifs de "haut niveau ", (2) les « espoirs » sportifs (aspirant ou international) et (3) les " partenaires d'entraînement ». Cette dernière catégorie regroupe des sportifs pratiquant une discipline en compétition, ne respectant pas les exigences fixées pour un des deux statuts précédents mais qui peuvent portant jouer un rôle essentiel dans la préparation d'un sportif de haut niveau et/ou d'un espoir sportif. Au sein de la Fédération Wallonie-Bruxelles, une cellule "Sport de haut niveau » avait déjà été créée en 2007. À l'heure actuelle, elle représente une aide précieuse pour les athlètes tout au long de leurs carrières sportive et professionnelle. Afin que tous les aspects soient pris en compte, cette cellule a été divisée en trois départements : la cellule " Aide à la performance sportive », la cellule «Projet de vie » et la cellule « Médico-sportive ».

La transition entre l'enseignement secondaire et le supérieur représente généralement une période charnière, associée à une intensification de la pratique sportive et une augmentation des difficultés scolaires (Wylleman, Alfermann \& Lavallée, 2004). Pour une population ordinaire, un peu plus d'un étudiant sur trois réussit sa première année dans le supérieur, soit entre 36 et $41 \%$ (Van Campenhoudt, Dell'Aquila \& Dupriez, 2008). Pour un étudiant qui combine ses études avec un sport pratiqué à haut niveau, la situation pourrait être encore moins favorable et expliquer les résultats obtenus par Squelard (2011). En effet, cet auteur a mis en évidence une diminution importante du nombre de sportifs sous contrat au-delà 


\section{eJRIEPS Spécial 2 (juillet 2019)}

des 18 ans, période charnière correspondant à l'entrée dans l'enseignement supérieur (tableau 1). Pour tenter de répondre à cette problématique de manière générale, Crépin et Demonty (2009) ont proposé cinq pistes d'action susceptibles de contribuer à la recherche de solutions concrètes :

- La réalisation d'un travail de fin d'études orienté sur un thème en lien avec les préoccupations du sportif ;

- Le décloisonnement des deux types d'enseignement (secondaire et supérieur) via une « école virtuelle »;

- Un travail commun sur les prérequis ;

- Une formation relative à la prise de notes ;

- Une immersion dans l'orientation choisie et parrainée par un autre étudiant.

Tableau I : recensement des sportifs reconnus par le Ministre des sports en Fédération WallonieBruxelles. Comparaison en fonction des âges en 2011-2012 (Squelard, 2011)

\begin{tabular}{|c|c|c|}
\hline Tranche d'âge & Total & Année de naissance \\
\hline$<12$ ans & 28 & 1998 \\
\hline 12 à 15 ans & 352 & $1996-1999$ \\
\hline 16 à 18 ans & 473 & $1993-1995$ \\
\hline 19 à 23 ans & 213 & $1988-1992$ \\
\hline 24 à 30 ans & 38 & $1981-1987$ \\
\hline 31 à 40 ans & 7 & $1971-1980$ \\
\hline$>40$ ans & 3 & $>1971$ \\
\hline Total & 1114 & \\
\hline
\end{tabular}

Dans les faits, Cloes et Polis (2005) ont constaté que les aménagements mis en œuvre dans les établissements supérieurs de la Fédération Wallonie-Bruxelles varient en fonction des institutions. À côté de cela, de nombreuses lacunes ont été relevées au niveau des structures offertes aux étudiants sportifs. Contrairement à ce qui est constaté dans certaines institutions françaises ou anglaises, ces derniers n'évoluent pas toujours dans les meilleures conditions (Cloes, Lenzen \& Polis, 2007). Cela expliquerait que beaucoup de jeunes sportifs envisagent de mettre un terme à leur carrière sportive lorsqu'ils arriveront dans le supérieur (Cloes, Schellings, Ledent \& Piéron, 2002). 


\section{eJRIEPS Spécial 2 (juillet 2019)}

\section{Questions de recherche}

Dans cette étude, notre objectif consiste à répondre aux questions et sous questions suivantes: (1) Quelle proportion de sportifs de haut niveau reconnus par la Fédération Wallonie-Bruxelles entame des études supérieures (Q1) ? De cette question découle une sous-question, à savoir, quel est leur taux de réussite (Q1a) ? (2) Comment les sportifs perçoivent-ils les aides disponibles (Q2) ? Cette question amène deux sous questions: Quels manquements et/ou difficultés ont-ils rencontrés (Q2a) ? Quelles suggestions formulent-ils dans la perspective d'améliorer le système (Q2b) ?

\section{Méthodologie}

\subsection{Population}

Pour mener à bien ce projet, nous avons signé une convention de partenariat avec l'AG Sport qui est responsable de l'octroi des statuts officiels des sportifs et qui nous a permis d'entamer un travail collaboratif. Des règles éthiques très strictes ont été établies afin de disposer des coordonnées des sportifs concernés (quel que soit leur titre). Dans le cadre de cette étude, réalisée en 2013-2014, nous nous sommes concentrés sur ceux ayant obtenu leur diplôme du secondaire entre 2010 et 2012. Le but était en effet d'interroger les sportifs après qu'ils ont potentiellement eu l'occasion de suivre une année d'études complète dans le supérieur. Au total, 352 sportifs figuraient dans la liste (68\% de garçons). II s'agissait de sportifs nés entre 1992 et 1995. Un questionnaire leur a été transmis par email avec, en préambule, les critères d'inclusion à notre recherche. Les personnes ne correspondant pas aux critères étaient directement informées qu'il n'était pas nécessaire de répondre au questionnaire. Au total, 126 réponses ont été récoltées; 103 réellement peuvent être exploitées, ce qui représente $34 \%$ de la population initiale. Les 23 questionnaires éliminés n'avaient pas été totalement complétés. Notre échantillon est constitué de 33 filles et 70 garçons.

\subsection{Outils}

Au vu de la population de départ $(n=352)$, nous nous sommes naturellement orientés vers un questionnaire. Créé au moyen du logiciel « Lime Survey », il est constitué de questions fermées avec une échelle d'évaluation de 1 à 10 , de questions à choix multiples et de questions ouvertes. Au total, 79 questions portant sur les aspects scolaire, sportif, social, relationnel, médical, organisationnel et ceux liés spécifiquement à la transition scolaire ont été posées. L'ensemble des items a été choisi sur base d'une analyse de la littérature (Hug, 


\section{eJRIEPS Spécial 2 (juillet 2019)}

2003 ; Javerlhiac, Bodin \& Robène, 2010 ; Javerlhiac, Leyondre \& Bodin, 2011 ; Wylleman et al., 2004). L'aval de l'AG Sport et de l'équipe de recherche a ensuite été nécessaire afin de finaliser le questionnaire et satisfaire aux exigences de chacun. Enfin, le questionnaire a été testé sur cinq anciens sportifs de haut niveau (hors critères de sélection pour notre étude), ce qui nous a permis de vérifier la bonne compréhension des questions et la pertinence des réponses collectées.

Afin de faciliter l'encodage et le traitement des données, le questionnaire a été placé en ligne. Le logiciel Lime Survey permet un transfert aisé des données vers les outils de traitement classiques tels qu'Excel et Statistica.

Les sportifs de haut niveau étant généralement très occupés par leurs activités sportives et scolaires, nous avons choisi de leur envoyer le lien URL du questionnaire par e-mail, ce qui leur laissait l'opportunité de le compléter à leur convenance. Le temps estimé pour compléter le questionnaire avoisine les vingt minutes. L'enquête s'est déroulée entre mars et mai 2014.

\subsection{Le traitement des données}

La qualité de la base de données Excel, élaborée à partir du logiciel Lime Survey, a été vérifiée. Lors du traitement des données, les questions ouvertes ont subi un codage catégoriel dont les fidélités inter (entre 86,6 et 95,5\%) et intra-analystes (entre 91,1 et $97,7 \%$ ) atteignent les pourcentages d'accords admis par la littérature. La partie du fichier contenant les réponses aux questions fermées a ensuite été transférée vers le logiciel Statistica (Statsoft, 2010) et a fait l'objet de statistiques élémentaires. Afin de comparer les différences obtenues pour les moyennes d'une même population à différents moments de leur parcours (études secondaires et études supérieures), nous avons réalisé des tests t de Student non appariés permettant de se prononcer sur la significativité ou non des résultats obtenus. À ce propos, une différence significative sera mise en évidence dans les figures à l'aide d'un astérisque lorsque que la valeur de $p$ est inférieure ou égale à 0,05.

\section{Résultats et discussion}

Au moment de la récolte des données, la majorité de nos sujets sont âgés de 20 ans, c'està-dire nés en 1994 (68\% de garçons), et ont effectué leur première année dans l'enseignement supérieur en 2012-2013. Ils proviennent de 55 établissements d'enseignement secondaire et de 15 institutions d'enseignement supérieur différents. Dans 


\title{
eJRIEPS Spécial 2 (juillet 2019)
}

la suite des résultats, nous présenterons les informations retenues en fonction des différents thèmes étudiés.

\subsection{Versant scolaire / versant sportif}

D’une manière générale, même si le taux de réussite scolaire atteint les $61 \%$ (figure 2), la transition scolaire (secondaire / haute école-université) semble vécue comme un véritable bouleversement. Ce pourcentage tient compte des réussites en juin (première session), en septembre (deuxième session) et des étudiants ayant acquis les 48 crédits sur 60 leur permettant de rejoindre l'année supérieure (crédits résiduels). Les résultats des sportifs de haut niveau de notre échantillon dépassent largement les 36\% à $41 \%$ de réussite mis en évidence par Van Campenhoudt et al. (2008) et Crepin et Demonty (2009), pour une population dite «classique », et rejoignent les constats de Deveen $(1971,1974)$ signalant que les sportifs de haut niveau réussissent en grande majorité leurs études. Nous resterons cependant très prudents par rapport à notre pourcentage élevé car, au sein de notre étude, il est possible que l'échec scolaire soit une cause de refus de participer à l'enquête.

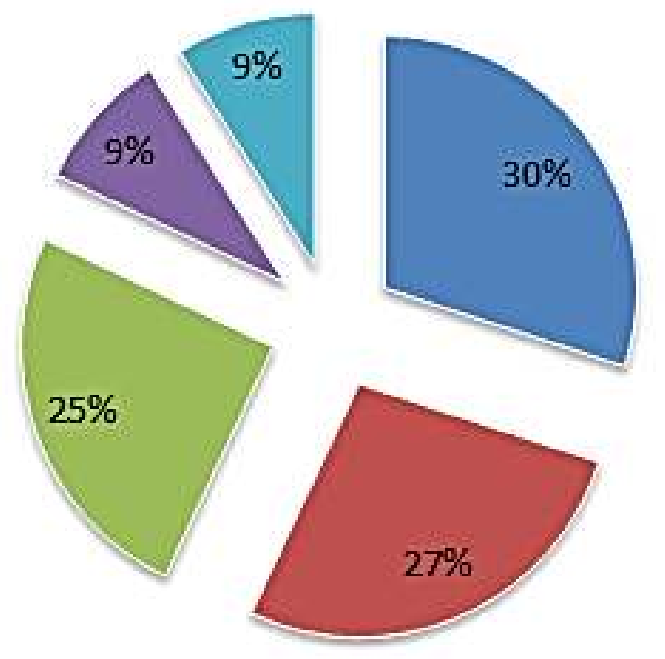

\author{
Echec \\ : Réussite en première \\ session \\ - Réussite en deuxième \\ session \\ abandon \\ Réussite avec crédit \\ résiduel
}

Figure 2 : résultats obtenus au terme de la $1^{\text {ère }}$ année dans le supérieur

À l'image de cet étudiant déclarant: "II m'était impossible de combiner études universitaires et haut niveau ! » (Q21), 9\% de notre échantillon ont pris la décision d'arrêter au cours de leur première année d'études supérieures. Les causes évoquées par ces sportifs sont doubles : (1) le manque de temps pour combiner les deux projets et (2) le manque de motivation à poursuivre un cursus dans le supérieur. Cela peut éventuellement s'expliquer par divers paramètres. À titre d'exemples, citons (1) l'absence d'un 


\section{eJRIEPS Spécial 2 (juillet 2019)}

aménagement horaire rendant la combinaison du sport et des études possible, (2) le manque de suivi du sportif, entraînant une diminution de sa motivation, ... Dans ces situations, le sportif est malheureusement incité à opérer un choix entre la poursuite de ses études ou de son sport de prédilection.

À l'inverse, $11 \%$ de l'échantillon a abandonné sa pratique sportive pour se consacrer aux études : "Je n'avais plus de temps pour continuer [mon sport] parce que j'ai commencé à l'université " (Q86). Ce constat va dans le sens des travaux menés par Polis \& Cloes (2005). Parallèlement, une forte diminution du nombre de statuts octroyés $(27 \%)$ est également relevée après cette année de transition. Comme le montre la figure 3 , les sportifs semblent avoir mis leur parcours sportif au second plan et, notamment, l'aspect compétitif où l'on observe une différence significative entre les enseignements secondaire et supérieur. Les sportifs ont donc tendance à réduire le temps consacré aux compétitions lors de leur entrée dans les études supérieures.

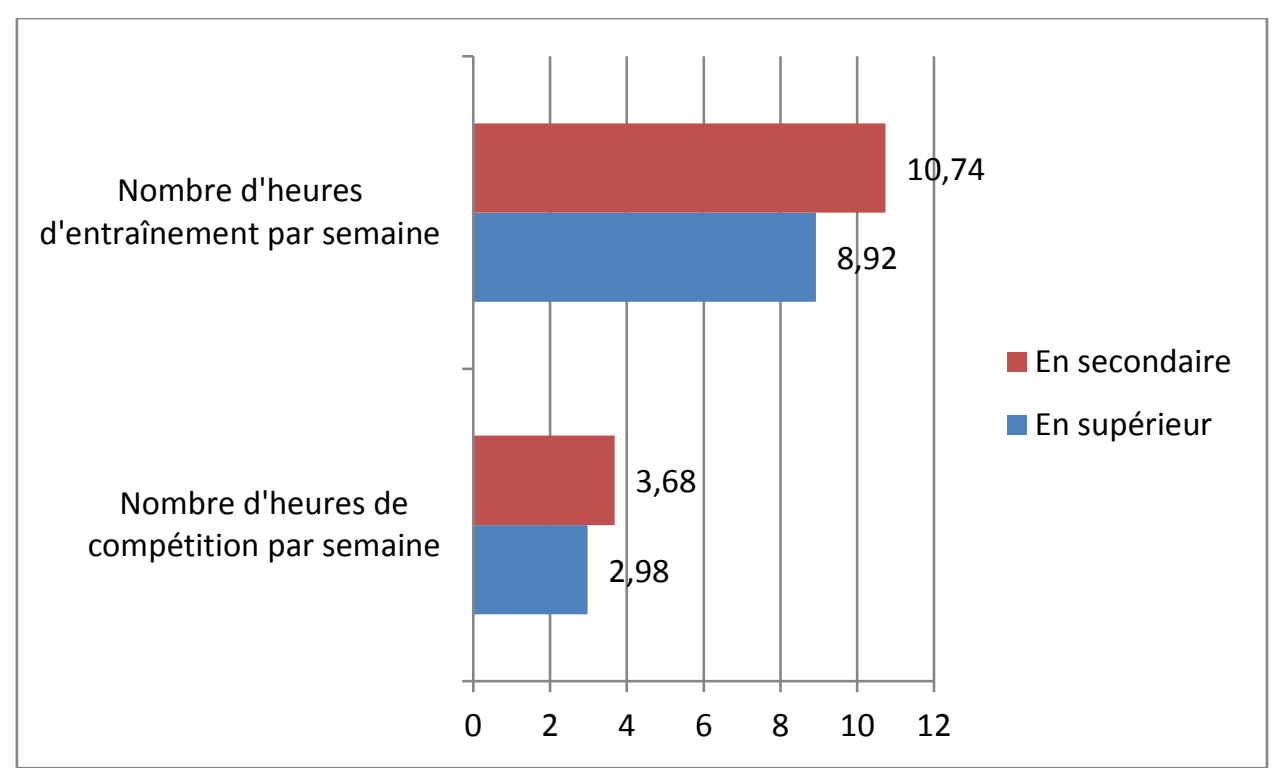

Figure 3 : moyenne du nombre d'heures d'entraînement et de compétition par semaine $(n=103)$

Si nous nous intéressons de plus près aux disciplines sportives pratiquées par les sportifs en fonction des résultats scolaires, nous constatons que les disciplines suivant le modèle «pseudo-professionnels » de Javherliac et al. (2011) obtiennent de meilleurs résultats. En effet, en Belgique, les revenus engendrés dans les disciplines telles que le judo, le volleyball, le hockey, la course d'orientation, le badminton, ... sont exceptionnellement suffisants que pour prétendre vivre de ses économies à la fin de sa carrière sportive. Cette perspective oblige les sportifs à penser de manière précoce à leur reconversion. À l'inverse, une discipline telle que le football s'inscrit dans une logique de filière professionnalisante et 


\section{eJRIEPS Spécial 2 (juillet 2019)}

semble rencontrer un taux de réussite scolaire moins important. À ce propos, il est opportun de préciser qu'un effet du genre intervient puisque $93,5 \%$ des sportifs pratiquant le football et interrogés au cours de notre étude sont des garçons.

Parmi les sujets, $84 \%$ estiment l'organisation du double projet plus simple en secondaire que dans le supérieur (figure 4). En effet, l'autonomie et l'investissement des étudiants sont bien plus importants en haute école ou à l'université par rapport à l'enseignement secondaire (De Knop \& Wylleman, 1998). Des questions complémentaires nous ont permis de mettre en évidence plusieurs disparités proposées à la figure 5. Le suivi du tuteur $(p=0,002)$, les moyens de rattrapage mis à disposition $(p=0,001)$ et les adaptations des horaires $(p \leq 0,001)$ sont les éléments qui sont les plus décalés et qui présentent des différences significatives entre le secondaire et le supérieur. L'existence de fortes disparités entre les établissements scolaires est également soulignée. Cette information est d'autant moins étonnante que les dispositions légales harmonisant les aménagements pour les sportifs de haut niveau en milieu scolaire n'existaient pas encore en 2010 (Squelard, 2011). Heureusement, elles tendent progressivement à se mettre en place depuis lors.

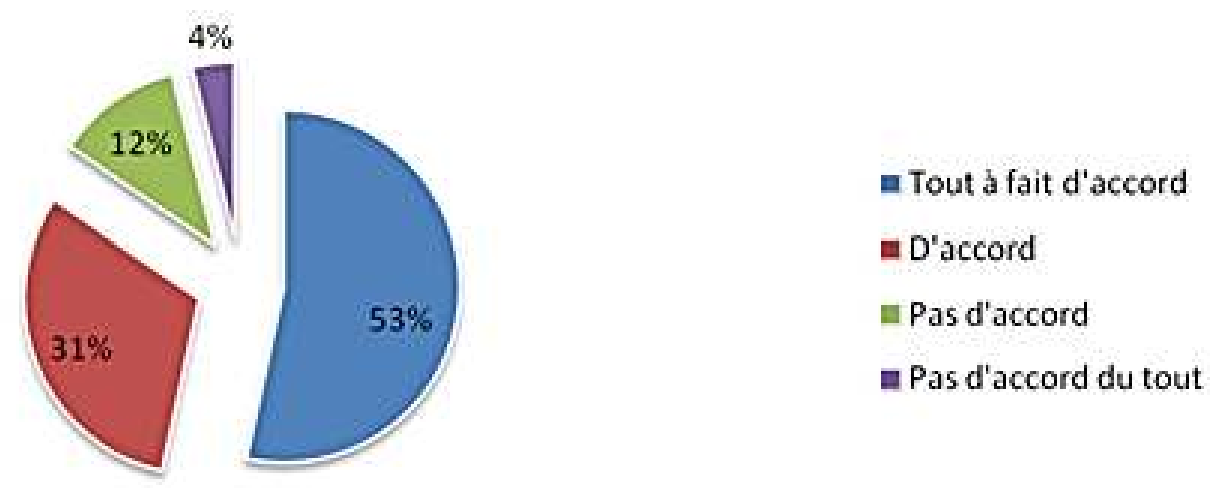

Figure 4 : avis des étudiants sportifs de haut niveau quant à la gestion du double projet ( $n=103)$ - Gérer sport et études est plus facile en secondaire que dans le supérieur.

Concernant les aménagements possibles au niveau scolaire pour réaliser au mieux leur double projet, $43 \%$ des sportifs signalent qu'ils n'ont pas reçu d'informations à ce sujet. Celles-ci étaient pourtant accessibles grâce à différents supports. Du côté de l'AG Sport, un regroupement des informations s'avèrerait manifestement utile pour offrir une meilleure visibilité des possibilités offertes aux sportifs de haut niveau. C'est peut-être également le rôle des fédérations sportives et des entraîneurs de pouvoir orienter leurs sportifs de haut niveau vers les informations utiles leur permettant de s'inscrire dans un projet de double carrière. À moins que les sportifs ne choisissent de se consacrer à leurs études, ce qui les écarterait des compétitions. Christensen et Sorensen (2009) rappellent l'importance du 


\section{eJRIEPS Spécial 2 (juillet 2019)}

contexte dans lequel évoluent les sportifs et, notamment, le rôle des clubs et des dirigeants qui peuvent sensibiliser positivement ces étudiants particuliers quant à l'intérêt d'obtenir une qualification académique en parallèle à la pratique sportive.

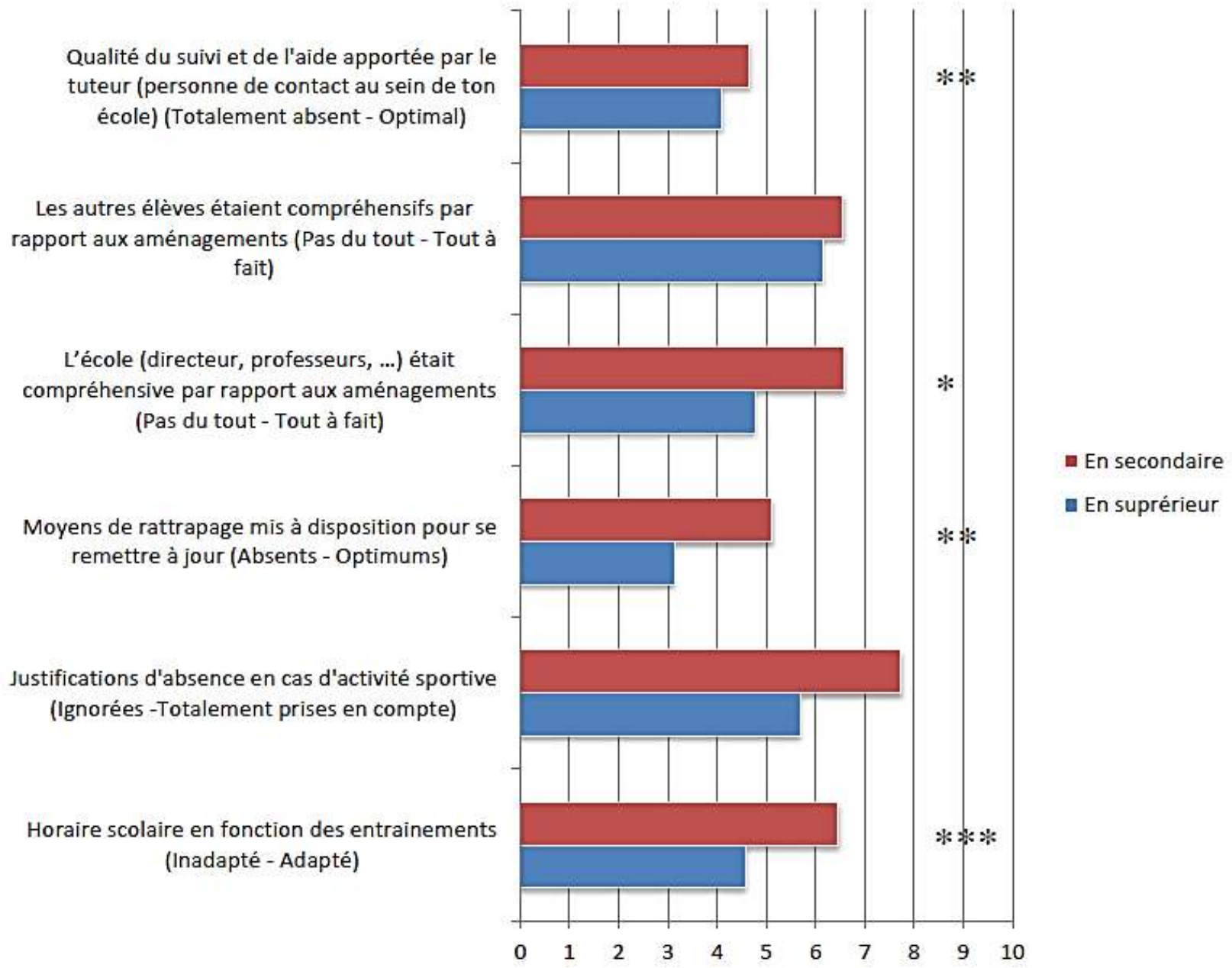

Figure 5 : avis des étudiants sportifs de haut niveau sur une échelle de 1 à 10 quant aux facteurs influençant leur parcours scolaire $(n=103)$

\subsection{Versant social / versant relationnel}

Sur le plan social, les sportifs se sentent mieux entourés dans l'enseignement secondaire. Les relations se dégradent quelque peu lors du passage vers le supérieur et des différences apparaissent entre ces deux milieux $(p \leq 0,001)$. Les études et le sport semblent cependant influencer positivement leur environnement familial (figure 6). En effet, nous pouvons aisément admettre que la pratique d'un sport de haut niveau d'un jeune fait la fierté de ses proches. La famille va donc se mobiliser autour du projet du sportif, principalement au niveau du secondaire. Forté (2006) parle de «bénéfices symboliques » partagés par l'athlète et sa famille. 


\section{eJRIEPS Spécial 2 (juillet 2019)}

Par ailleurs, devenir sportif de haut niveau demande un sacrifice et un investissement important. Combiné à un parcours scolaire, cela ne peut qu'engendrer un phénomène d'isolement constaté par Cloes et al. (2002). Malheureusement, nous ajoutons à cela le peu de relations établies avec les autres sportifs de haut niveau. Nous pensons toutefois que les échanges entre sportifs de haut niveau pourraient exercer une influence positive sur la gestion du double projet et sur le partage des bonnes pratiques.

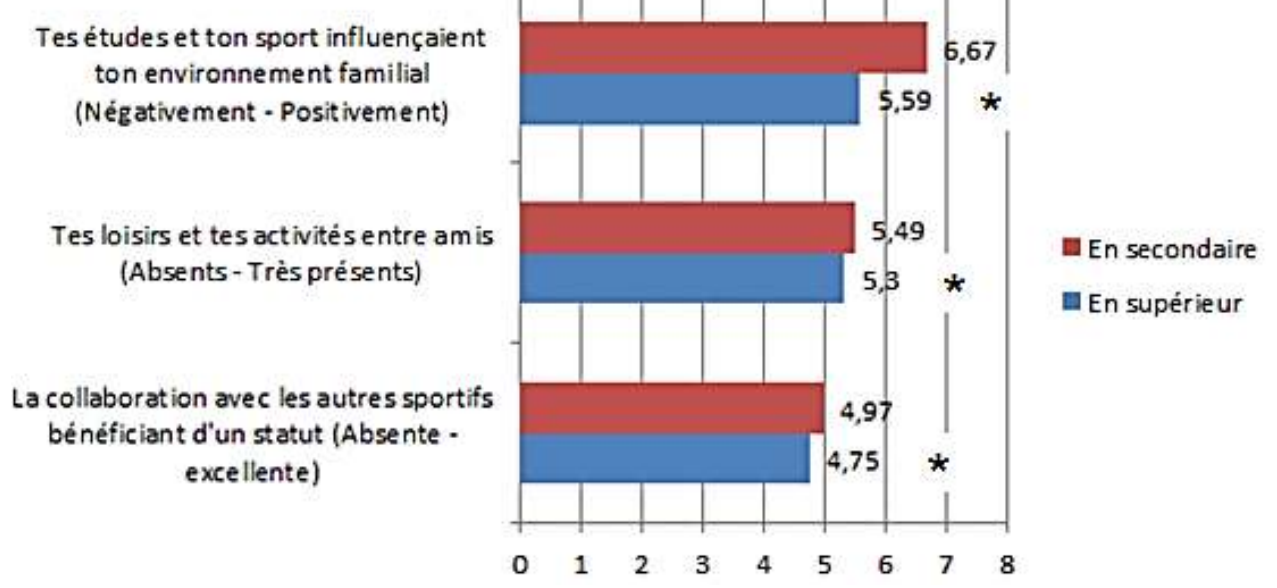

Figure 6 : avis des sujets quant aux aspects sociaux

La fréquence des loisirs et des activités entre amis est assez faible chez les sportifs sous contrat. Elle s'approche de la note moyenne des $5 / 10$. Le constat est encore plus médiocre lors du passage dans le supérieur $(p \leq 0,001)$. De Knop, Wylleman, Van Hoeke et Bollaert (1999) avaient déjà attiré l'attention sur le fait qu'un environnement social perturbé pouvait entraîner les sportifs dans un cercle vicieux à propos de leur gestion du temps. En approfondissant le sujet, nous constatons que les sportifs qui émettent les avis les plus positifs pour cet item sont ceux qui se perfectionnent dans une discipline collective (70\%). Il est vraisemblable que leurs loisirs soient partagés en grande partie avec les membres de l'équipe, ce qui induit une plus grande fréquence des activités entre amis, et donc un score plus positif à la question posée. Dans une étude qui s'intéresse à des athlètes de haut niveau en France, Forté (2006) souligne qu'une articulation entre les milieux amical et sportif permettrait de "diminuer le stress lié aux ambitions sportives et prévenir des sentiments de saturations sources de démotivation » chez certains sportifs (p.63).

Si les connexions entre le milieu sportif et le milieu scolaire sont mentionnées comme très peu présentes par nos sujets, les aides proposées par les différentes structures (l'enseignement secondaire, le supérieur et l'AG Sport) sont en revanche évaluées comme efficaces, même si les relations avec l'AG Sport semblaient trop ponctuelles en 2011-2012. 


\section{eJRIEPS Spécial 2 (juillet 2019)}

Afin de réduire le fossé entre le milieu scolaire et le milieu sportif, les fédérations sportives et/ou les clubs pourraient faire preuve de davantage d'initiative et inviter, par exemple, des classes dans lesquels évoluent les sportifs sous contrat à des compétitions qui se déroulent au sein d'une structure proche de l'établissement scolaire, organiser des journées portes ouvertes et/ou des initiations pour les élèves/étudiants...

\subsection{Santé}

Dans le domaine de la santé, le manque de suivi au niveau alimentaire nous paraît intéressant à soulever. De manière générale, même si aucune différence significative n'apparaît entre les deux niveaux d'enseignement, les valeurs obtenues dans notre étude atteignent des moyennes très faibles (4,4 en secondaire et 4,3 en supérieur). Ce résultat est inquiétant car, si l'on se réfère aux travaux de Filaire, Rouveix, Bouget et Pannafieux (2007), le risque de présenter des troubles du comportement alimentaire (TCA) est plus important chez les sportifs que chez les sédentaires. En comparant les avis en fonction du sexe, les filles mentionnent un résultat moyen $(3,9 / 10)$ plus négatif que celui des garçons $(4,6 / 10)$, sans différence significative entre les deux. La population féminine mériterait tout de même une attention particulière à ce niveau afin d'éviter les dérives liées aux TCA. Du côté des disciplines pratiquées, les données pourraient être en accord avec la littérature puisque, soumis à des contraintes de poids, les judokas semblent être davantage pris en charge sur le plan alimentaire dans le supérieur $(7,6 / 10)$ que dans le secondaire $(5,5 / 10)$. Les catégories de poids rencontrées fin du secondaire et début de supérieur correspondent aux catégories «juniors » et « seniors ». À cette période de la vie, la croissance du sportif est déjà bien avancée, le maintien d'un poids déterminé est donc plus important que dans les catégories «jeunes ». Salmi, Pichard et Jousselin (2010) attirent l'attention sur les difficultés à diagnostiquer des TCA car les variations de poids sont assez courantes dans le milieu sportif et les répercussions sur la santé ne sont pas toujours immédiates. Les TCA peuvent être réactionnels, chroniques, psychiatriques,... La boulimie est plus difficile encore à détecter. En réalité, tous les sportifs sont susceptibles d'être touchés par cette problématique. Un suivi plus régulier de chaque sportif s'avère donc essentiel.

D'une manière générale, les périodes de repos et de sommeil sont perçues comme à peine suffisantes chez les sportifs de haut niveau avec une dégradation significative lors du passage en supérieur ( $p=0.02$ ). L'intensification de l'entraînement combinée à une matière à étudier plus importante pourrait engendrer un stress supérieur et expliquer en partie ce phénomène. Petersen et Nittinger (2003) avaient également classé le manque de sommeil 


\section{eJRIEPS Spécial 2 (juillet 2019)}

ainsi que le manque de qualité au niveau des repas dans une liste des agents stressants pour les sportifs de haut niveau.

Dans l'ensemble, tant avant qu'après la période de transition, les sportifs considèrent être peu impactés par l'apparition de blessures, même si une différence significative existe entre les deux types d'enseignement $(p<0.001)$. Le suivi médical des sportifs ainsi que les activités de prévention semblent donc de qualité. Par rapport au constat formulé en 2005 par Polis et Cloes, cet aspect semble s'être considérablement amélioré. Actuellement, des accès privilégiés vers les soins de santé (médecins, psychologues, kinésithérapeutes, ...) ainsi que le suivi des tests à l'effort semblent porter leurs fruits. Dans le même ordre d'idées, les difficultés liées aux blessures ont été prises en compte dans une étude de Piffaretti (2006), en Suisse. Les élèves inscrits dans des sport-études en danse éprouvent davantage de difficulté à gérer la transition secondaire obligatoire (12-15 ans) vers le secondaire postobligatoire (15-19 ans) que des danseuses inscrites précédemment dans des classes traditionnelles. Les élèves des classes sportives expliquent leur abandon par la difficulté de concilier sport et études dans le post-obligatoire ou en évoquant une fatigue psychologique et des blessures (Piffaretti, 2006). Le suivi médical et la prévention de plus en plus présents dans le sport de haut niveau s'avèrent donc cruciaux pour tenter d'assurer la longévité de la carrière de l'athlète.

Ayant étudié la santé des sportifs de haut niveau à l'INSEP à Paris, Salmi, Pichard et Jousselin (2010) mettent en garde contre les effets pervers du sport à haute intensité. D'après ces auteurs « le sport ne crée pas la pathologie, il s'agit plutôt d'une rencontre entre une personnalité, un sport et un contexte donné qui crée les conditions d'émergence de la pathologie » (p.1). Une analyse du contexte plus général (alimentation, sommeil, variations de poids, état de forme,...) ressenti par l'athlète parait pertinente afin de déceler la moindre faille du système mis en place et y remédier aussi rapidement que possible.

\subsection{Versant organisationnel}

Même si les sportifs les jugent acceptables, il existe une différence significative entre le ressenti des sportifs par rapport aux conditions vécues en termes de déplacements entre les deux niveaux scolaires. En effet, dans de nombreux cas, le club et l'établissement scolaire secondaire sont géographiquement proches du domicile du sportif. À l'inverse, lors du passage en supérieur, les distances augmentent et il est fréquent de constater que les étudiants se détachent du logement familial (Wylleman et al., 2004). La figure 7 montre clairement une autonomie plus importante des sportifs en supérieur. Les parents sont plus 


\section{eJRIEPS Spécial 2 (juillet 2019)}

difficilement en mesure de les accompagner. Une distance plus importante à parcourir ainsi que la possibilité d'obtenir le permis de conduire à 18 ans sont deux hypothèses plausibles pour expliquer ce constat. En Allemagne, Diehl et Hilger (2016) constatent que bon nombre d'étudiants « ordinaires » éprouvent des difficultés à surmonter les changements de mode de vie imposés par la transition entre les deux niveaux d'études. Cela se manifesterait concrètement par une réduction de l'activité physique. Cette situation s'avère d'ailleurs très logiquement accentuée lorsqu'il y a un changement de lieu de résidence.

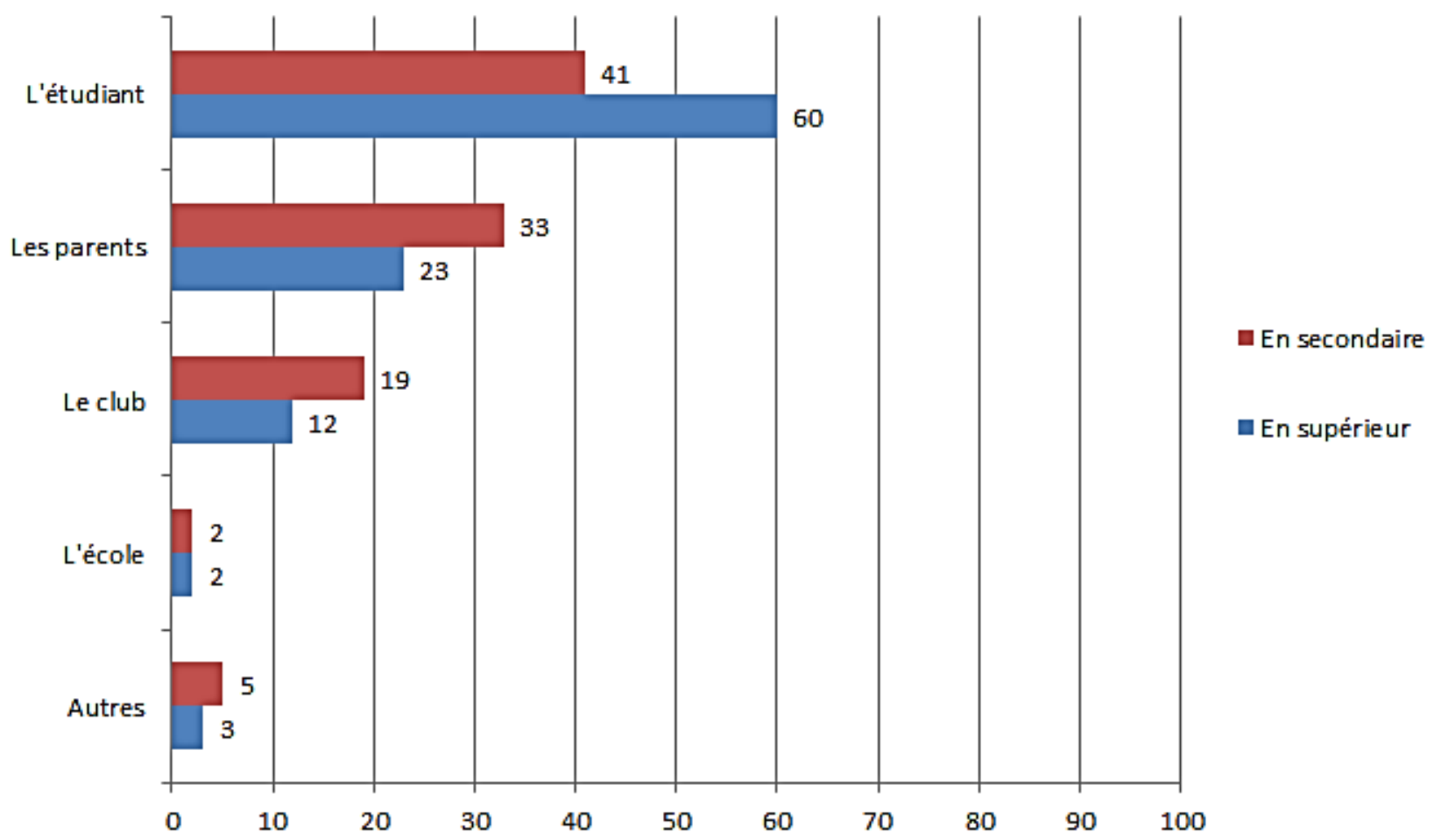

Figure 7 : prise en charge du transport (\% de réponses pour 103 sujets)

\subsection{Aspects divers}

En dernière année du secondaire, les opportunités les plus appréciées par les sportifs de haut niveau sont: (1) la possibilité de participer à des compétitions $(36,6 \%)$; (2) la possibilité de justifier ses absences scolaires grâce au statut ; (3) l'opportunité d'être dispensés de certains cours pour participer à des activités sportives $(23,7 \%)$ et ; (4) l'aménagement possible des horaires de cours en fonction de la pratique sportive. Un faible pourcentage des répondants $(6,9 \%)$ estime que le statut ne leur a pas été utile dans le secondaire. II est bien évident qu'en fonction des disciplines considérées, les sportifs ne rencontrent pas les mêmes besoins.

Dans le cadre des études supérieures (figure 8), avec 27,3\%, la possibilité de participer à des compétitions reste une des opportunités les plus appréciées: "J'ai pu participer aux 


\section{eJRIEPS Spécial 2 (juillet 2019)}

$J O$ ! » (Q51). À côté de cela, le même pourcentage d'étudiants mentionne que le statut ne leur a été d'aucune utilité dans le supérieur. En approfondissant ce résultat interpellant, nous constatons que plus de $80 \%$ des étudiants partageant cet avis sont issus d'une haute école (permettant d'obtenir un diplôme de bachelier, soit 180 crédits). Cela signifierait peutêtre que les lacunes dans les aménagements proposés dans le supérieur non universitaire, mises en évidence par Polis et Cloes (2005), seraient toujours d'actualité. Cela renforce à nouveau le constat de disparités entre les institutions d'enseignement.

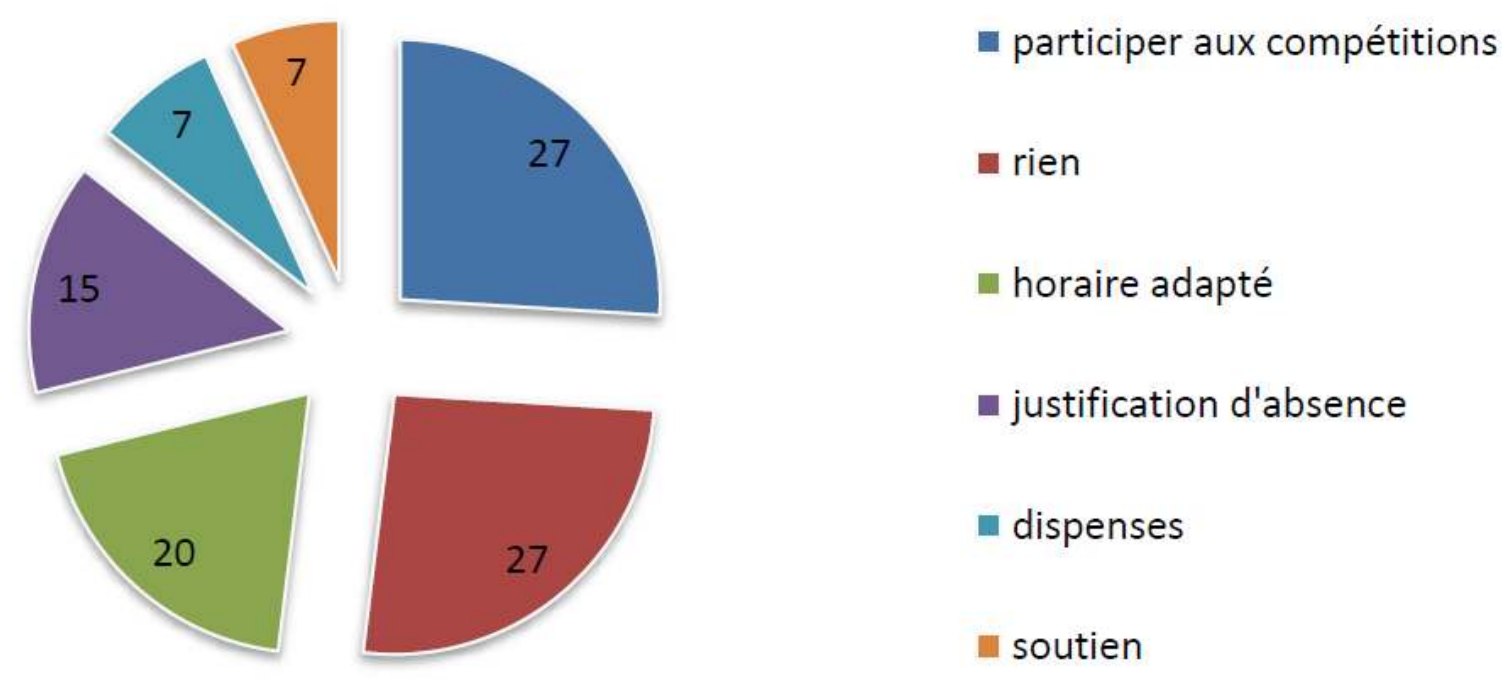

Figure 8 : éléments les plus positifs dans le supérieur $(n=99)$

En ce qui concerne les éléments perçus comme négatifs lors de la dernière année des études secondaires, la gestion du temps est l'item le plus souvent mis en évidence $(25,3 \%)$. Pour $23,3 \%$ des étudiants, rien ne leur a semblé compliqué. Dans ce cas, soit ils ont bénéficié de tous les aménagements souhaités, soit ils n'en n'ont tout simplement pas eu besoin pour réussir leur double parcours. En secondaire, 18,2\% des sportifs mentionnent déjà des difficultés à participer à des activités sociales et de loisir : "Enchainer une journée complète de cours puis prendre les transports en commun jusqu'à l'entraînement et, seulement, rentrer le soir pour commencer les devoirs... " (Q48). À l'image de cet étudiant, nous pouvons aisément comprendre la difficulté de ces sportifs à gérer leur temps et à trouver des créneaux disponibles pour d'autres activités.

Au cours de la première année dans le supérieur, l'élément le plus souvent cité est à nouveau la difficulté à gérer son temps $(27,3 \%)$. Étant donné le caractère récurent du problème et ce, dans les deux types d'enseignement, une formation sur le sujet mériterait d'être proposée aux sportifs, voire étendue à l'ensemble des élèves, qu'ils soient sportifs ou 


\section{eJRIEPS Spécial 2 (juillet 2019)}

non. L'absence d'éléments négatifs est soulignée par $20 \%$ des sportifs tandis que le manque de souplesse des horaires de cours est soulevé comme problématique par 16,2\% de la population.

La figure 9 indique clairement que $73 \%$ des étudiants pensent que les facilités octroyées à ceux qui bénéficient d'un statut sont mieux aménagées dans le secondaire que dans le supérieur. Les difficultés vécues au cours de la période de transition sont de natures diverses (figure 10). L'élément le plus souvent cité est le manque de soutien dans la double carrière $(32,8 \%)$. Le passage d'un établissement à un autre implique un changement de tuteur. Les démarches administratives pouvant prendre un certain temps, le sportif risque de devoir se prendre en charge pendant une période plus ou moins prolongée. II serait intéressant à ce niveau d'assurer une continuité du suivi des sportifs de haut niveau en proposant des tuteurs non attachés à un établissement mais directement à une cellule de I'AG Sport ou à une fédération sportive.
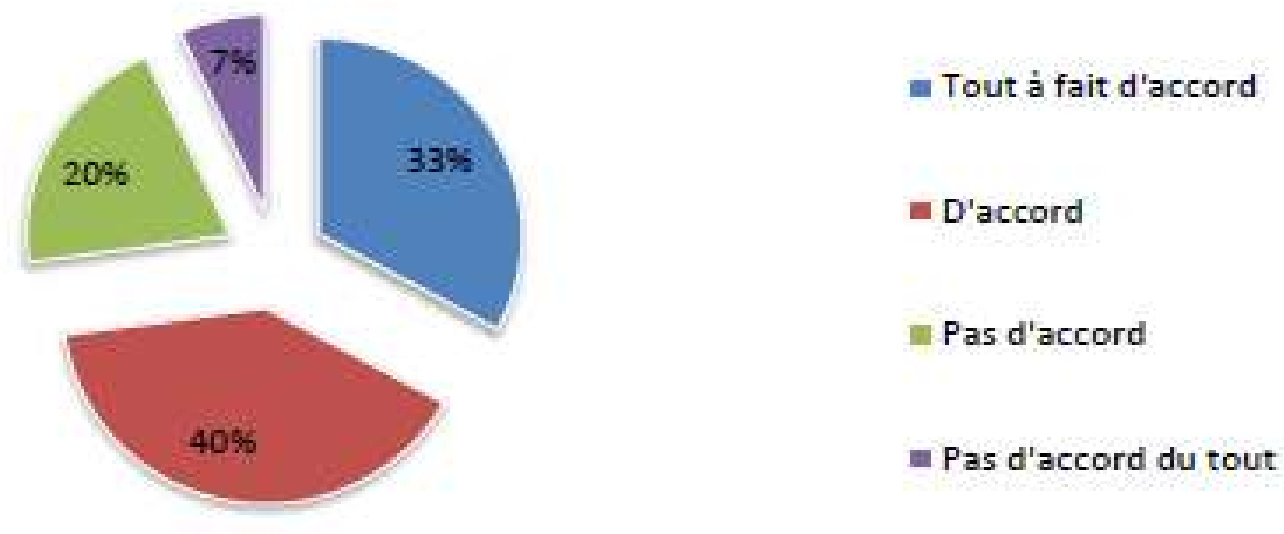

Figure 9 : avis des étudiants quant aux facilités octroyées par le statut de sportif - Les facilités sont mieux aménagées en secondaire que dans le supérieur.

La quantité de matière importante à intégrer représente une autre difficulté à surmonter $(27,4 \%)$. Le nouvel environnement à apprivoiser représente lui aussi un obstacle pour $16,4 \%$ d'entre eux et la combinaison des horaires scolaires et sportifs cause du souci à $15 \%$ des sujets interrogés.

Au cours de la période de transition, 58 étudiants (56\%) affirment avoir reçu des informations sur la gestion du double projet des sportifs de haut niveau. Malgré l'énergie que cela représente au niveau temporel et organisationnel, la forme de transmission la plus souvent soulevée est l'entretien direct (31\%). La consultation de sites internet revient également fréquemment (29\%). Le site de l'AG Sport ( $\underline{w w w . a d e p s . b e)}$ ) semble très clair à ce niveau. Celui de l'enseignement en Fédération Wallonie-Bruxelles 


\title{
eJRIEPS Spécial 2 (juillet 2019)
}

(www.enseignement.be) propose lui aussi toute une série d'informations utiles pour les sportifs bénéficiant du statut. Les e-mails et les flyers remportent moins de suffrages (13,8\% chacun). Un résultat étonnant est celui des activités de promotion des études. En effet, seulement $8,5 \%$ des sportifs disent avoir reçu des informations via ce créneau. Proposer des stands spécifiques aux étudiants sportifs serait peut-être une idée à exploiter.

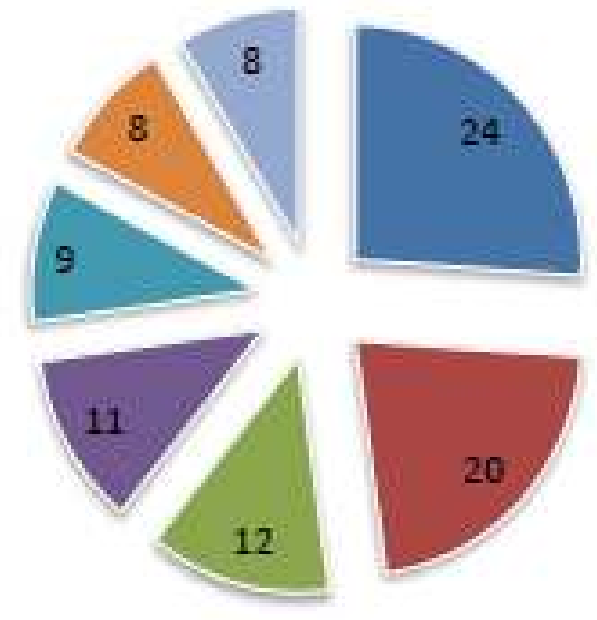

\author{
- Absence de soutien \\ - Trop de matière \\ in Nouvel environnement \\ - Combinaison des horaires \\ Pas de difficultés \\ Gestion des déplacements \\ Gestion du temps
}

Figure 10 : difficultés vécues lors de la transition $(n=73)$

\subsection{Pistes d'amélioration}

À la fin du questionnaire, les sportifs de haut niveau étaient invités à nous faire part de suggestions d'amélioration afin de pouvoir rechercher des pistes d'action adaptées aux besoins des principaux intéressés pour gérer plus aisément la transition scolaire. La plus fréquemment citée concerne l'amélioration de l'accompagnement $(42,5 \%)$. Une meilleure communication entre les différents acteurs, c'est-à-dire entre les milieux entourant l'élève arrive en seconde position (19\%). Des réunions permettant d'informer et d'échanger des informations à propos du sportif concerné pourraient s'avérer utiles pour s'approcher du modèle de Hug (2003). À ce propos, un des outils proposés par la cellule «Projet de vie » de l'AG Sport est le CEL (Carnet d'Entraînement et de Liaison). Il a pour objectif d'améliorer les interactions entre les différents protagonistes en lien avec le sportif (parents, staff du centre de formation, staff en club, enseignants, ...). Le transfert de cet outil papier en version online est souhaité par trois sportifs de haut niveau. Les informations pourraient ainsi être accessibles à tout moment et par toutes les personnes concernées. Notons également que plus de $15 \%$ de la population souhaiteraient recevoir davantage d'informations sur les aménagements possibles au sein des différentes institutions d'enseignement. 


\section{Limites de l'étude}

Une première limite est en lien avec la taille de notre échantillon. Nous avons en effet pu récolter l'avis de 103 sujets sur une liste de 352 potentiels. Même si cela représente près d'un tiers de la population, nous ne pouvons tirer de conclusions précises en fonction des disciplines sportives qui se retrouvent très nombreuses dans cette étude.

Ensuite, nous n'avons pas tenu compte de l'orientation professionnelle future des étudiants. Ces derniers ne sont pas à l'abri d'un choix ne correspondant pas à leur attente ou d'un choix orienté pour concorder davantage avec la pratique sportive en cours.

\section{Perspectives de recherche}

Pour donner suite à l'analyse des résultats, la création d'un portail informatisé réservé aux sportifs de haut niveau nous parait indispensable. L'idée serait de s'inspirer de l'INSEP en France qui dispose déjà d'un portail de suivi quotidien des sportifs. Ce dernier aurait pour objectif de rassembler toutes les informations concernant les sportifs (activités scolaires, sportives et quotidiennes) sur un seul média et partagé entre tous les acteurs. En combinant les informations contenues dans le CEL avec le soutien d'une plate-forme, un outil adapté aux sportifs francophones pourrait être mis sur pied. En effet, comme le mentionne l'Agence Wallonne des Télécommunications (2013), les nouvelles technologies vont modifier les modes de relations de nos sociétés en proposant un environnement numérique global qui peut être décrypté selon quatre axes :

- Les technologies «Mobiles » et «Web 2.0 ». Ceux-ci favorisent une fusion des mondes réel et virtuel ;

- Le «cloud computing ", une plate-forme ubiquitaire de services qui est accessible par tous et partout ;

- La capacité à gérer un grand nombre de données en les individualisant ;

- Une redistribution des rôles permettant aux individus de mieux agir sur leur environnement (social, professionnel, ...).

Une analyse des statistiques récoltées directement via ce portail pourrait également nous aiguiller vers d'éventuelles futures études.

Pour enrichir et mieux comprendre les résultats obtenus, il nous parait pertinent d'analyser, dans un futur proche, la qualité des aménagements véritablement proposés par les établissements scolaires et académiques. 


\section{eJRIEPS Spécial 2 (juillet 2019)}

\section{Conclusion}

Malgré les évolutions dans le domaine pour aider les sportifs de haut niveau, la transition entre les études secondaires et supérieures représente toujours une véritable période charnière. Même si la majorité d'entre eux optent pour un double projet de vie et obtiennent des résultats satisfaisants, certains sportifs doivent opérer un choix : sport ou études ! D'après les résultats obtenus, $11 \%$ des sportifs de notre échantillon ont mis de côté leur sport afin de se consacrer à leurs études. Inversement, $9 \%$ ont choisi de mettre leurs études de côté afin de consacrer tout leur temps au sport. Soulignons tout de même la grande proportion de réussite (61\%) pour cette population particulière en première année dans le supérieur.

D’après nos sujets, les aménagements dont les étudiants sportifs peuvent bénéficier sont perçus plus positivement dans le secondaire que dans le supérieur. La quantité et la complexité de la matière abordée ainsi que la plus grande autonomie dans le supérieur semblent être des paramètres jouant un rôle influant dans le succès ou l'échec de cette transition. La gestion du temps et les déplacements semblent également plus complexes à gérer. De plus, le manque d'information perçu par les sportifs concernant les aménagements possibles ne leur facilite pas la tâche.

Dans les manquements mis en évidence, les sportifs citent, entre autre, le manque de soutien des différentes structures dans leur double projet (établissements de formation, AG Sport, ...), le manque de suivi de l'alimentation et les déficiences de communication entre les intervenants. Les disparités entre les établissements de formation et les deux niveaux d'enseignement sont toujours d'actualité.

Les propositions principales que nous formulons portent sur (1) le développement d'un outil informatique rassemblant les informations utiles aux sportifs de haut niveau, combiné à un outil d'échanges entre les différents protagonistes entourant le sportif et (2) un accompagnement unique et prolongé entre les deux types d'enseignement.

\section{Bibliographie}

Administration Générale du Sport (n.d.). De la renaissance de l'éducation physique à la cellule projet de vie en passant par l'émergence du sport et la naissance de l'ADEPS. Travaux internes non publiés par l'Administration Générale du Sport.

Agence Wallonne des Télécommunications (2013). Quatre axes pour comprendre notre futur numérique. Consulté le 4 août 2014:

http://www.awt.be/web/awt/index.aspx?page=awt,fr,r12,100,per\#ancre0 


\section{eJRIEPS Spécial 2 (juillet 2019)}

Association Suisse des Entraîneurs (2004). Concepts de l'ASE. Consulté le 5 décembre 2013 sur Internet : http://sport-etude.ecolevs.ch/concept.asp?inside=concept

Cloes, M. (2012). La Wallonie en mouvement : vers une culture sportive et de l'activité physique ? In M. Germain et R. Robaye (Eds.), L'état de la Wallonie. Portrait d'un pays et de ses habitants - 2011 (pp. 70-80). Namur : Les éditions namuroises.

Cloes, M., Lenzen, B., \& Polis, A. (2007). Analysis of the support provided to studentathletes in Wallonia. The case of higher education. In, J.A. Diniz, F. Carreiro da Costa \& M. Onofre (Eds.), Active lifestyles: The impact of education and sport. Proceedings of the AIESEP 2005 World Congress (pp. 85-92). Lisbonne: Faculdade de Motricidade Humana. Available on Internet: http://hdl.handle.net/2268/40305

Cloes, M. \& Polis, A. (2005). Analyses des structures mises en place à l'intention des sportifs de haut niveau dans l'enseignement supérieur. Comparaison des différentes régions et pays. Université de Liège, Belgique. Consulté le 26 janvier 2017 sur Internet : http://hdl.handle.net/2268/13514

Cloes, M., Schelings, V., Ledent, M., \& Piéron, M. (2002). Sport-études: comparaison des caractéristiques motivationnelles et des relations sociales des élèves sportifs et de leurs condisciples. eJRIEPS, 1, 57-72.

Crepin, F., \& Demonty, I. (2009). Etude portant sur l'amélioration de la transition entre l'enseignement secondaire et l'enseignement supérieur en Communauté Française de Belgique par la mise en œuvre d'expériences pilotes associant des enseignants du troisième degré de l'enseignement secondaire et de l'enseignement supérieur. Université de Liège, Belgique. Consulté le 9 mai 2018 à l'adresse suivante: enseignement.be/download.php?do id=5780

Christensen, M. K., \& Sorensen, J. K. (2009). Sport or school? Dreams and dilemmas for talented young Danish football players. European Physical Education Review, 15 (1), 115-133.

De Bosscher, V., De Knop, P., van Bottenburg, M., Bingham, J. \& Shibli, S. (2006). Sports Policy Factors Leading To International Sporting Success. Consulté le 26 janvier 2017 sur Internet : http://www.vub.ac.be/SBMA/spliss

De Knop, P. \& Wylleman, P. (1998). De combinatie van studie en topsport in een maatschappelijke, organisatorische en onderzoekscontext. Nieuw Tijdschrift van de Vrije Universiteit Brussel, 2, 69-95.

De Knop, P., Wylleman, P., Van Hoecke, J. \& Bollaert, L. (1999). Sports management - A European approach to the management of the combination of academics and elite- 


\section{eJRIEPS Spécial 2 (juillet 2019)}

level sport. In S. Bailey (Ed.), Perspectives - the interdisciplinary series of Physical Education and Sport Sciences. Vol. 1. School sport and competition (pp 49-62). Oxford : Meyer \& Meyer Sport.

Diehl, K., Hilger, J. (2016). Physical activity and the transition from school to university: a cross-selectional survey among university students in Germany. Sciences et Sport, 31 (4), 223-226.

Filaire, E., Rouveix, M., Bouget, M. \& Pannafieux, C. (2007). Prévalence des troubles du comportement alimentaire chez le sportif. Sciences et Sport, 22 (3-4), 135-142. Consulté le 26 janvier 2017 sur Internet : http://www.sciencedirect.com/science/article/pii/S0765159707000408

Forté, L. (2006). Fondements sociaux de l'engagement sportif chez les jeunes athlètes de haut niveau, Movement \& sport Sciences, 59 (3), 55-67.

Hug, P-A. (2003). Evaluation de la filière gymnasiale sport-études du Canton de Vaud. Lausanne.

Javerlhiac, S., Bodin, D. \& Robène, L. (2010). Préparer sa reconversion, entre engagement personnel et contraintes sportives. Terrains \& travaux, 17, 75-91.

Javerlhiac, S., Leyondre, F., \& Bodin, D. (2011). Sportifs de haut niveau et double projet. Entre bonne intention et faisabilité. International journal of violence and school, 12, 26-58.

Petersen, C. \& Nittinger, N. (2003). Fit to play tennis: High performance training tips (first edition). Vancouver : CPC Physio corp.

Piffaretti, M. (2006). Abandon du sport de compétition chez les jeunes : Étude longitudinale dans les Classes "sport et danse" du Canton de Genève (Résumé de la thèse de doctorat en sciences sociales et politiques non publiée). Université de Lausanne, Suisse. Consulté le 17 mai 2018 sur Internet : http://www.actsport.ch/content db/content/reussiteabandonsport20061.pdf

Polis, A. \& Cloes, M. (2005). Analyse des structures mises en place à l'intention des sportifs de haut niveau de l'enseignement supérieur. Comparaison dans différentes régions et pays. Liège : Université de Liège, Département des Sciences de la Motricité. Consulté le 20 août 2015 sur Internet : http://hdl.handle.net/2268/13514

Salmi, M., Pichard, C. \& Jousselin, E. (2010). Psychopathologie et sport de haut niveau. Science et Sports, 25 (1), 1-10. 
eJRIEPS Spécial 2 (juillet 2019)

Squelard, V. (2011). Premiers éléments d'un audit du sport en Fédération Wallonie Bruxelles. Travaux internes non publiés par l'Administration Générale du Sport. Bruxelles.

Van Campenhoudt, M., Dell'Aquila, F., \& Dupriez, V. (2008). La démocratisation de l'enseignement supérieur en Communauté française de Belgique : état des lieux. Les cahiers de la recherche en éducation et formation, 65, 1-41. Consulté sur Internet : https://halshs.archives-ouvertes.fr/halshs-00561927

Wylleman, P., Alfermann, D. \& Lavallée, D. (2004). Career transitions in sport : European perspectives. Psychology of Sport and Exercise, 5 (1), 7-20. 